\title{
Body composition but not cardiorespiratory fitness correlate with adipokines in overweight-obese adults
}

\begin{abstract}
Propose: To correlate parameters regards to body composition, cardiorespiratory fitness and adipokines in overweight-obese adults.

Methods: A cross-sectional study of subjects with overweight-obese $(n=30)$ was conducted. Anthropometric data, peak oxygen consumption, levels of serum leptin, adiponectin, ghrelin, insulin and glucose were assessed. Insulin resistance was defined by the index HOMA-IR and a ratio adiponectin/leptin was calculated.

Results: No correlations between cardiorespiratory fitness were observed which hormones or adipokines. However, when correlated anthropometric parameters, we observed a significant correlation between leptin and hip circumference $(\mathrm{r}=0.459$; $\mathrm{p}=0.021)$, leptin and fat mass $(\%)(\mathrm{r}=0.705 ; \mathrm{p}=0.001)$ an inverse correlation between ghrelin and ratio $\mathrm{w} / \mathrm{h}(\mathrm{r}=-0.518 ; \mathrm{p}=0.007)$ and ratio adiponectin/leptin which fat mass $(r=-0.701 ; p=0.001)$.
\end{abstract}

Conclusion: In obese individuals, body composition parameters but not functional capacity, was correlated with elevated levels of adipokines.

Keywords: sedentary, inflammation, obesity
Volume 2 Issue 2 - 2015

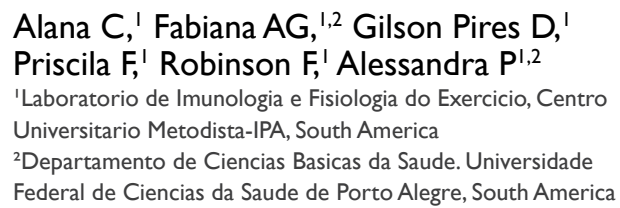

Correspondence: Alessandra Peres, Universidade Federal de Ciencias da Saude de Porto Alegre, Rua Sarmento Leite, 245-Centro Historico, Porto Alegre, Rio Grande do Sul, Brazil, Tel 555। 33161227,Email peres@ufcspa.edu.br

Received: October 29, 2014 | Published: April 07, 2015
Abbreviations: AC, abdominal circumference; BMI, body mass index; HC, hip circumference; HOMA-IR, homeostatic model assessment-insulin resistance; $\mathrm{VO}_{2 \text { Peak }}$, peak of oxygen consumption; WC, waist circumference; WHO, world health organization; ELISA, enzyme-linked immunosorbent assay; HR, heart rate; SD, standard deviation

\section{Introduction}

Overweight and obesity defined as body mass index (BMI) greater than $25 \mathrm{~kg} / \mathrm{m}^{2}$ for overweight and $30 \mathrm{~kg} / \mathrm{m}^{2}$ for obesity is a world-wide epidemic. ${ }^{1}$ Inactive lifestyle leads to the expansion of adipose tissue and it is accompanied by infiltration of immune cells and an increase in the release of adipokines is a condition known as systemic lowgrade inflammation, characterized by a two-to-four fold elevation of proinflammatory mediators when compared to healthy lean

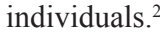

Certain adipokines, such leptin and ghrelin have important functions in the control of food intake and inflammation, but obese individuals present high levels of these adipokines and low interaction with their receptors, which causes a reduction in the perception of satiety and increased pro-inflammatory effects. ${ }^{3}$ On the other hand, adiponectin is synthesized inversely to fat mass and it has been postulated it improves anti-inflammatory effects, insulin sensitivity and protect against cardiovascular diseases. ${ }^{2,3}$

Cardiorespiratory fitness induces important effects in the control of low-grade inflammation and individuals with higher levels of oxygen consumption have lower levels of circulating pro-inflammatory cytokines. ${ }^{4}$ But the relationship between Cardiorespiratory fitness and adipokines in obesity is unclear. ${ }^{5,6}$ Thus, the objective of this study is to correlate parameters regarding body composition, cardiorespiratory fitness and adipokines in overweight-obese adults. Our hypothesis is that overweight-obesity individuals show a positive correlation between oxygen consumption and adiponectin and an inverse correlation with inflammatory adipokines.

\section{Methods}

This is a cross-sectional study. We recruited by convenience overweight and obese adult 30 volunteers ( $>18$ years) of both sexes from the city of Porto Alegre, Brazil. The inclusion criteria for the patients were: sedentary, BMI between $25-39.9 \mathrm{~kg} / \mathrm{m}^{2}$, over 18 years old, both genders and medical certificate approving the practice of physical training. All patients participating in this study gave their informed written consent. This study was approved by the Ethics Committee of Centro Universitario Metodista IPA of Porto Alegre, Brazil (058/2012)

The study was development in steps and split in two days: on day one, individuals arrived to the Laboratory of Research Center at 7:008:00am. In a overnight fast to collect the fasting blood sample and assess of body composition. Three days after the first visit, individuals returned to the laboratory for the cardiopulmonary test in a previously scheduled time. The experimental design was developed so adipokine concentrations were considered in basal condition and were not influenced by the treadmill test.

Body weight and height were determined by a semi-analytical scale (Welmy, Sao Paulo, Brazil). Body composition was assessed by the bioelectrical impedance method (Byodinamics BIA 310e), respecting all pre-test guidelines, considering the values of fat mass percentage (\%). We also measured waist (WC), abdomen (AC) and hip circumferences $(\mathrm{HC})$. 
Serum leptin (DBC Leptin ELISA kit, USA), adiponectin (MBL Adiponectin ELISA kit, USA), Ghrelin (Millipore Corporation, USA) and fasting insulin (DRG Insulin ELISA kit, USA) were analyzed in duplicate, using the methodology of Enzyme-Linked Immunosorbent Assay (ELISA). Fasting blood-glucose was determined by colorimetric method (COBAS ${ }^{\circledR} \mathrm{C} 111$, Bayer, USA). Blood-glucose and insulin data were used to calculate the Homeostasis Model of Insulin Resistance (HOMA-IR): [fasting insulin $(\mu \mathrm{UI} / \mathrm{mL}) \times$ fasting glucose $(\mathrm{mmol} / \mathrm{L}) / 22.5]$.

The peak oxygen uptake $\left(\mathrm{VO}_{2 \text { Peak }}\right)$ test was conducted on a motorized treadmill (ATL Inbramed Millennium, Porto Alegre, Brazil) and to collect gases we used an ergospirometry (V02000, Medgraphics, St. Paul, USA). Heart rate (HR) was monitored during the test using a frequency counter (Polar FT7, USA). $\mathrm{VO}_{2 \text { Peak }}$ was defined as the highest value obtained after treatment of the data by three blind (or independent) researchers. Verbal encouragement was provided for all subjects to ensure maximum values were reached.

Descriptive statistics were presented as mean and standard deviations (SD). The Shapiro-Wilk test was used to assess the normality of the distribution of variables. Correlation between variables and other clinical conditions were performed using Pearson's correlation. The level of significance was set in $p \leq 0.05$. All the statistical analysis was performed with SPSS (SPSS for Windows 20.0).

\section{Results}

Body composition, functional capacity and biochemical variables were presented in Table 1. The mean of the BMI was defined as obesity grade one $\left(34,14 \pm 3,94 \mathrm{~kg} / \mathrm{m}^{2}\right)$, lower $\mathrm{VO}_{2 \text { Peak }}(27.05 \pm 5.36 \mathrm{ml}$. kg. $\left.\mathrm{min}^{-1}\right)$ and a higher levels of leptin $(79.84 \pm 23.59 \mathrm{ng} / \mathrm{mL})$ and ghrelin $(52.64 \pm 39.62 \mathrm{ng} / \mathrm{mL})$ and showed no resistance insulin rating according to reference values for the Brazilian population. ${ }^{7}$

Table 2 shows the data of the correlations between circulating adipokines with body composition and cardiorespiratory fitness. While no significant results were found in any variable of cardiorespiratory with adipokine correlations $(p<0.05)$, the ratio adiponectin/leptin was correlated with body mass $(p=0.03)$, waist circumference $(p=0.03)$ and an inverse percentage correlation of fat mass $(\mathrm{p}=0.00)$. Leptin was correlated with hip circumference $(\mathrm{p}=0.02)$ and percentage of fat mass $(\mathrm{p}=0.00)$ and ghrelin shows a significant inverse correlation with the waist/hip ratio ( $\mathrm{p}=0.00)$. Blood-glucose, insulin and HOMA-IR index do not show significant correlation with either variable.

Table I Clinical and biochemical variables of individuals with overweightobesity

\begin{tabular}{|c|c|}
\hline & Mean \pm SD \\
\hline Gender M/F & $10: 20$ \\
\hline Age (years) & $48.96 \pm 10.50$ \\
\hline Body mass (kg) & $91.22 \pm 15.56$ \\
\hline BMI $\left(\mathrm{kg} / \mathrm{m}^{2}\right)$ & $34.14 \pm 3.94$ \\
\hline$W C(\mathrm{~cm})$ & $99.21 \pm 8.01$ \\
\hline $\mathrm{AC}(\mathrm{cm})$ & $105.65 \pm 8.84$ \\
\hline $\mathrm{HC}(\mathrm{cm})$ & $115.60 \pm 8.24$ \\
\hline Ratio Waist/Hip & $0.82 \pm 0.18$ \\
\hline Fat Mass (\%) & $38.45 \pm 3.89$ \\
\hline $\mathrm{VO}_{2 \text { Peak }}(\mathrm{L} / \mathrm{min})$ & $2.46 \pm 0.66$ \\
\hline $\mathrm{VO}_{2 \text { Peak }}\left(\mathrm{ml} . \mathrm{kg} \cdot \mathrm{min}^{-1}\right)$ & $27.05 \pm 5.36$ \\
\hline $\mathrm{RER}_{\max }$ & $1.00 \pm 0.89$ \\
\hline $\mathrm{HR}_{\text {max }}(\mathrm{bpm})$ & $146.46 \pm 22.90$ \\
\hline Adiponectin (ng/mL) & $35.55 \pm 4.01$ \\
\hline Ghrelin (ng/mL) & $52.64 \pm 39.62$ \\
\hline Leptin $(\mathrm{ng} / \mathrm{mL})$ & $79.84 \pm 23.59$ \\
\hline Ratio Adip/Lep & $0.49 \pm 0.20$ \\
\hline Insulin $(\mu \mathrm{UI} / \mathrm{mL})$ & $9.58 \pm 2.49$ \\
\hline Glucose (mg/dL) & $98.39 \pm 12.59$ \\
\hline HOMA-IR & $2.44 \pm 0.79$ \\
\hline
\end{tabular}

SD, standard deviation; BMI, body mass index; WC, waist circumference; $A C$, abdominal circumference; HC, hip circumference; RER, respiratory exchange ratio; HR, hear rate

Table 2 Correlations between adiponectin, leptin, ghrelin and ratio adiponectin/leptin with parameters of cardiorespiratory fitness in overweight-obese adults

\begin{tabular}{|c|c|c|c|c|c|c|c|c|}
\hline & \multicolumn{2}{|c|}{ Adiponectin (ng/mL) } & \multicolumn{2}{|c|}{ Leptin (ng/mL) } & \multicolumn{2}{|c|}{ Ghrelin (ng/mL) } & \multicolumn{2}{|c|}{ Ratio Adipo/Lep } \\
\hline & $\mathbf{r}$ & $\mathbf{p}$ & $\mathbf{r}$ & $\mathbf{p}$ & $\mathbf{r}$ & $\mathbf{p}$ & $\mathbf{r}$ & $\mathbf{p}$ \\
\hline $\mathrm{VO}_{2 \text { Peak }}$ Absolute (L/min) & 0.235 & 0.25 & -0.183 & 0.37 & 0.174 & 0.39 & 0.305 & 0.13 \\
\hline $\mathrm{VO}_{2 \text { Peak }}(\mathrm{ml} . \mathrm{kg} \cdot \mathrm{min})$ & 0,105 & 0.61 & -0.17 & 0.93 & 0.029 & 0.88 & 0.05 & 0.81 \\
\hline RER & 0.182 & 0.38 & 0.023 & 0.91 & -0.063 & 0.76 & 0.062 & 0.77 \\
\hline $\mathrm{HR}_{\operatorname{Max}}(\mathrm{bpm})$ & 0.17 & 0.416 & 0.191 & 0.349 & -0.061 & 0.76 & -0.112 & 0.59 \\
\hline Body mass (kg) & 0.226 & 0.27 & -0.267 & 0.18 & 0.312 & 0.12 & 0.431 & 0.03 \\
\hline BMI $\left(\mathrm{kg} / \mathrm{m}^{2}\right)$ & 0.144 & 0.49 & 0.113 & 0.58 & 0.158 & 0.44 & 0.222 & 0.91 \\
\hline$W C(\mathrm{~cm})$ & 0.322 & 0.11 & -0.302 & 0.134 & 0.117 & 0.57 & 0.435 & 0.03 \\
\hline$A C(\mathrm{~cm})$ & -0.039 & 0.85 & -0.108 & 0.6 & 0.216 & 0.28 & 0.159 & 0.44 \\
\hline $\mathrm{HC}(\mathrm{cm})$ & 0.298 & 0.15 & 0.433 & 0.02 & 0.252 & 0.22 & -0.237 & 0.26 \\
\hline Ratio Waist/Hip & -0.081 & 0.69 & 0.185 & 0.36 & -0.652 & 0 & -0.369 & 0.061 \\
\hline Fat Mass (\%) & -0.252 & 0.22 & 0.678 & 0 & 0.003 & 0.98 & -0.689 & 0 \\
\hline
\end{tabular}

Pearson's Correlation: $p$ values were considered significant when $\leq 0.05$ (in bold). 


\section{Discussion}

Obesity is characterized by low grade chronic inflammation, primarily by desbalance between pro- and anti-inflammatory adipokines. The excess of fat mass is the major cause for this condition. However, some theories suggest that functional capacity can also influence it. $^{2}$ The major findings of this study are: a) adipokines are not correlated with the variables of cardiorespiratory fitness in overweight-obese individuals; b) leptin, ratio adiponectin/leptin and ghrelin present a correlation with some variables of central obesity; c) others hormones such as cortisol and insulin and a HOMA-IR are not correlated to any of the variables.

Leptin is produced proportionally to total fat mass and obese individuals have hyper-leptinemia, which results in a chronic inflammatory state. ${ }^{8}$ Our results demonstrate that in addition to a correlation between leptin and body fat percentage, a moderate correlation was found to hip circumference and it demonstrates a clear relationship with central obesity. The inverse correlation between ghrelin and waist/hip ratio may be show that obese individuals present lower levels and production of ghrelin when compared to lean subjects. $^{9}$

The parameters of body composition also correlate with adiponectin/leptin ratio, a marker of unbalance between pro and anti-inflammatory adipokines. This ratio is directly associated with clinical and biochemical parameters of insulin resistance, becoming a better marker when compared to the isolated use of serum leptin or adiponectin. ${ }^{10}$

Due to fact that cardiorespiratory fitness (here related to $\mathrm{VO}_{2 \text { peak }}$ ) is a factor for reduction of inflammation in obese individuals, ${ }^{11}$ may be these variables seem to correlate with adipokines. Contrary to our hypothesis adiponectin, leptin or ghrelin are not correlated with oxygen consumption. In part, these results demonstrate that the proinflammatory adipokines are related to body mass and that may be the regulation of these adipokines through exercise training could be dependent on the reduction of body fat and not the increase of the cardiorespiratory capacity. ${ }^{6}$ Contrary to our results, a study with 250 Brazilian army men showed a positive correlation between $\mathrm{VO}_{2 \text { Peak }}$ and adiponectin. However, it is important to note that when the authors made adjustments according to the BMI of individuals, this correlation disappears. ${ }^{12}$

Elevated adipokines, especially leptin have a close relationship with cardiovascular risk factors ${ }^{13}$ and cardiovascular mortality. ${ }^{14}$ Nevertheless, the lack of correlation between cardiovascular fitness and adipokines in our study may indicate these inflammatory markers can have secondary deleterious effects in cardiovascular health and not a direct effect. This theory falls along with the study of RubioGuerra and coworkers (2013), who demonstrated a relationship between circulating adipokines with carotid intima-media thickness, a marker associated with increase of myocardial infarction and stroke.

\section{Conclusion}

In conclusion, although there are theories about healthy obese subjects, ${ }^{15}$ a strong relationship between abdominal obesity and inflammatory adipokines - as demonstrated in this study - should be taken into consideration. Finally, we do not ignore the possibility that the lack of changes observed in the present study may be partly explained by the relatively small sample size.

\section{Acknowledgements}

None.

\section{Conflict of interest}

The author declares no conflict of interest.

\section{References}

1. World Health Organization (WHO). Global Health Observatory, Obesity. World Health Organization; 2013.

2. Denis GV, Obin MS. Metabolically healthy obesity:origins and implications. Mol Aspects Med. 2013;34(1):59-70.

3. Ouchi N, Parker JL, Lugus JJ, et al. Adipokines in inflammation and metabolic disease. Nat Rev Immunol. 2011;11(2):85-97.

4. Rana JS, Nasir K, Santos RD, et al. Increased level of cardiorespiratory fitness blunts the inflammatory response in metabolic syndrome. Int $J$ Cardiol. 2006;110(2):224-230.

5. Hosick PA, McMurray RG, Cooper DM. The relationships between leptin and measures of fitness and fatness are dependent upon obesity status in youth. Pediatr Exerc Sci. 2010;22(2):195-204.

6. Nassis GP, Papantakou K, Skenderi K, et al. Aerobic exercise training improves insulin sensitivity without changes in body weight, body fat, adiponectin, and inflammatory markers in overweight and obese girls. Metabolism. 2005;54(11):1472-1479.

7. Geloneze B, Vasques AC, Stabe CF, et al. HOMA1-IR and HOMA2IR indexes in identifying insulin resistance and metabolic syndrome: Brazilian Metabolic Syndrome Study (BRAMS). Arq Bras Endocrinol Metabol. 2009;53(2):281-287.

8. Jung CH, Kim MS. Molecular mechanisms of central leptin resistance in obesity. Arch Pharm Res. 2013;36(2):201-207.

9. Cummings DE, Weigle DS, Frayo RS, et al. Plasma ghrelin levels after diet-induced weight loss or gastric bypass surgery. $N$ Engl $J$ Med. 2002;346(21):1623-1630.

10. Inoue $\mathrm{M}$, Yano $\mathrm{M}$, Yamakado $\mathrm{M}$, et al. Relationship between the adiponectin-leptin ratio and parameters of insulin resistance in subjects without hyperglycemia. Metabolism. 2006;55(9):1248-1254.

11. Poelkens F, Eijsvogels TM, Brussee P, et al. Physical fitness can partly explain the metabolically healthy obese phenotype in women. Exp Clin Endocrinol Diabetes. 2014;122(2):87-91.

12. Martinez EC, Fortes MS, Anjos LA. Influence of nutritional status and VO2max on adiponectin levels in men older than 35 years. [corrected]. Arq Bras Cardiol. 2011;96(6):471-476.

13. Nakamura K, Fuster JJ, Walsh K. Adipokines: a link between obesity and cardiovascular disease. J Cardiol. 2014;63(4):250-259.

14. Kizer JR. Adiponectin, cardiovascular disease, and mortality:parsing the dual prognostic implications of a complex adipokine. Metabolism. 2014;63(9):1079-1083.

15. Ortega F, Lee D, Katzmarzyk P, et al. The intriguingly metabolically healthy but obese phenotype:cardiovascular prognosis and role of fitness. Eur Heart J. 2013;34(5):389-397. 